ZWISCHEN TRADITION UND

INNOVATION: KARL VON DEN

STEINENS VÖLKERKUNDLICHE

FORSCHUNGEN IM XINGU-

QUELLGEBIET (1883-1887)

UND IHRE BEDEUTUNG FÜR DIE

DEUTSCHE AMERIKANISTIK DES

19. JAHRHUNDERTS ${ }^{1}$

\author{
Sandra Rebok \\ CSIC, Área de Cultura Científica, Madrid
}

srebok@orgc.csic.es

\author{
ENTRE LA TRADICIÓN Y LA \\ INNOVACIÓN: LA INVESTIGACIÓN \\ ANTROPOLÓGICA DE KARL VON \\ DEN STEINEN EN LA REGIÓN \\ DE LAS FUENTES DEL XINGÚ \\ (1883-1887) Y SU SIGNIFICADO \\ PARA EL AMERICANISMO \\ ALEMÁN DEL SIGLO XIX
}

RESUMEN: Este artículo analiza los estudios antropológicos llevados a cabo por el viajero y científico alemán Karl von den Steinen a finales del siglo XIX en la región del río Xingú en Brasil Central. En primer lugar se ofrece una breve introducción sobre las dos expediciones por él dirigidas así como sobre los intereses científicos que las motivaron. A continuación se contrapone la orientación ideológica y temática del americanismo de la época con la investigación de Karl von den Steinen en Brasil, con objeto de ver en qué sentido ha trabajado siguiendo las pautas metodológicas habituales de su época y en qué aspectos ha abandonado ese marco tradicional a fin de desarrollar innovaciones en la investigación. Tras este análisis de la contribución de von den Steinen al desarrollo del americanismo alemán, el artículo se dedica a contestar la pregunta de hasta qué punto sus estudios son aún de relevancia para nosotros hoy día, y si su metodología y resultados siguen siendo válidos en la actualidad. Finalmente, se presentan los vínculos de su orientación ideológica en relación con los de sus predecesores y sucesores, a fin de detectar quién pudo influir en Karl von den Steinen $y$, a la inversa, quién pudo imbuirse de sus ideas.

PALABRAS CLAVE: Historia de la ciencia, expediciones, Karl von den Steinen, antropología, Brasil, americanismo, río Xingú.
ZUSAMMENFASSUNG: Dieser Artikel analysiert die anthropologischen und ethnologischen Studien, die der deutsche Reisende und Wissenschaftler Karl von den Steinen Ende des 19. Jahrhunderts in der Region des Flusses Xingu in Zentralbrasilien durchgeführt hat. Einleitend wird zunächst eine kurze Übersicht gegeben über beide von inm geleitete Forschungsreisen und ihre jeweiligen wissenschaftlichen Anliegen. Anschließend wird die ideologische bzw. inhaltliche Ausrichtung der damaligen Amerikanistik der Brasilienforschung Karl von den Steinens gegenübergestellt, um aufzuzeigen, in welcher Hinsicht er in der zeitgenössischen Tradition gearbeitet und in welchen Aspekten er diesen Rahmen verlassen und Innovationen in der Forschung herbeigeführt hat. Nach der Analyse des Beitrags von den Steinens zur Entwicklung der deutschen Amerikanistik befasst sich dieser Artikel mit der Frage, inwiefern seine damalige Forschung für uns heute noch relevant ist bzw. ob seine Methodik und deren Resultate weiterhin Gültigkeit haben. Abschließend erfolgt eine Darstellung der Verbindung seiner ideologischen Ausrichtung und denen seiner Vorgänger bzw. Nachfolger, um so zu erkennen, von wem Karl von den Steinen beeinflusst wurde und umgekehrt, an wen er seine Ideen weitergegeben hat.

SCHLÜSSELWÖRTER: Wissenschaftsgeschichte, Forschungsreisen, Karl von den Steinen, Ethnologie, Brasilien, Amerikanistik, Rio Xingu. 


\title{
BETWEEN TRADITION AND INNOVATION: THE ANTHROPOLIGICAL INVESTIGATION OF KARL VON DEN STEINEN IN THE REGION OF THE XINGÚ HEADWATERS (1883-1887) AND THEIR SIGNIFICANCE FOR THE GERMAN AMERICANISM OF THE 19TH CENTURY
}

\begin{abstract}
This essay attempts to analyze the anthropological and ethnographical studies realized by the German traveller and scientist Karl von den Steinen at the end of the 19th century in the region of the river Xingu in Central Brazil. First a short overview will be given of both expeditions directed by him and their scientific concerns. Then the ideological and thematical orientation of the americanism of this epoch will be contrasted with the research undertaken by Karl von den Steinen in Brazil, in order to illustrate the ways in which he worked in the tradition of his time and in which aspects he departed from this framework and effected innovations in his research. After this analysis of the contributions of Karl von den Steinen to the development of German americanism, this article focuses on the question of, to which point his studies are still relevant today and if his methodology and its results continue to be valid in our time. Finally, the relationship of his ideological orientation with those of his predecessors and his successors will be thematized in order to explore Karl von den Steinen's influences and whom he, in turn, influenced.
\end{abstract}

Key words: History of science, expeditions, Karl von den Steinen, anthropology, Brazil, Americanism, Rio Xingu.

In der folgenden Untersuchung werden die Zusammenhänge herausgearbeitet, die die ethnologische Forschung einer Epoche mit dem zu dieser Zeit vorherrschenden Geistesleben sowie den Zuständen und Ereignissen des politischen und gesellschaftlichen Seins verbinden. Diesem Anliegen liegt die These zugrunde, dass bei der völkerkundlichen Forschung nicht nur die Entdeckung einer fremden Kultur selbst, sondern ihre Wahrnehmung durch die eigene Gesellschaft sowie die Aufnahme in diese mindestens ebenso bedeutsam ist. Diese Wahrnehmung ist sowohl subjektiv als selektiv, denn hinsichtlich der erforschten Völker werden nur diejenigen Aspekte festgehalten, die überhaupt wahrgenommen werden können aufgrund der Prägung durch bzw. den ständigen Vergleich mit der eigenen Kultur, der äußeren Zwänge sowie der Interessen, unter denen der Kontakt zu der anderen Kultur hergestellt wird und nicht zuletzt aufgrund der Erwartungen, die zu Hause an die Berichte der Forscher gestellt werden. Die Begegnung mit fremden Völkern wird dadurch beeinflusst, dass das Eigene und Vertraute in das Fremde und Unvertraute projiziert und somit das Fremde mit den eigenen Kategorien wahrgenommen und schließlich an den eigenen Normen und Werten gemessen wird (Gewecke, 1992, 12). Die Art der Beschreibung des Fremden bzw. der Vermengung mit dem Eigenen gibt demnach oft mehr von den in der eigenen Tradition verwurzelten Stereotypen wieder, als dass es als reales Abbild der fremden Wirklichkeit verstanden werden sollte. Der zeitgeschichtliche Kontext und die in inm dominierenden wissenschaftlichen sowie auch ideologischen Fragestellungen und Interessen geben demnach die Rahmenbedingungen der ethnologischen Forschung vor und prägen so auch die inhaltliche und methodische Ausrichtung der deutschen Amerikanistik.

Aufgezeigt und eingeordnet in den historisch-ethnologischen Gesamtzusammenhang werden diese Thesen am Bei- 
spiel Karl von den Steinens und seiner Brasilienforschung im Quellgebiet des Flusses Xingu (Thieme, 1993; Rebok, 2002, 371-393). In den 80er Jahren des vergangenen Jahrhunderts nahm die Erforschung der Ethnien im bis dahin unbekannten Quellgebiet des Xingu, einem rechten Nebenfluss des unteren Amazonasstromes, einen wichtigen Platz in der völkerkundlichen Forschung in Deutschland ein (Schaden, 1993). Diese wiederum wurde durch den deutschen Wissenschaftler Karl von den Steinen eingeleitet, indem er in den Jahren 1883 zum ersten und 1887 zum zweiten Mal eine Expeditionsreise in das genannte Gebiet unternommen hat. Sowohl durch die im Anschluss daran veröffentlichten Bücher und Artikel als auch durch die Ermutigung und Förderung seiner Nachfolger, hat Karl von den Steinen die völkerkundliche Erforschung dieser Gegend Brasiliens maßgeblich bestimmt.

Einleitend wird zunächst eine kurze Übersicht gegeben über beide von ihm geleiteten Forschungsreisen in das Xingu-Gebiet und ihre jeweiligen wissenschaftlichen Anliegen. Um sein Engagement für die völkerkundliche Forschung besser in den Kontext einordnen zu können, widmet sich das nächste Kapitel dem biografischen und persönlichen Hintergrund von den Steinens.

Anschließend wird der Bezug hergestellt zwischen der dargestellten Makro- und der Mikroebene, also der Zusammenhang zwischen der ideologischen bzw. inhaltlichen Ausrichtung der damaligen Amerikanistik und der Brasilienforschung Karl von den Steinens. Bei dieser Gegenüberstellung soll herausgearbeitet werden, welche Aspekte der Makroebene auch in der Mikroebene auftauchen, in welcher Hinsicht er also in der zeitgenössischen Tradition gearbeitet und in welchen Aspekten er diesen Rahmen verlassen und Innovationen in der Forschung herbeigeführt hat. Hierdurch soll auch die Bedeutung und Besonderheit seiner Forschung verdeutlicht werden.

Nach der Analyse des Beitrags von den Steinens zur Entwicklung der deutschen Amerikanistik stellt sich die Frage, inwiefern seine damalige Forschung für uns heute noch relevant ist bzw. ob seine Methodik und deren Resultate weiterhin Gültigkeit haben.

Ein weiterer wichtiger und bedeutsamer Aspekt zur Einordnung seiner Person in die geschichtliche Entwicklung ist nicht nur die Darstellung seiner ideologischen Ausrichtung, sondern die Verbindung zwischen diesen Ansätzen und denen seiner Vorgänger sowie seiner Nachfolger. Somit wird in diesem Zusammenhang abschließend der Fragen nachgegangen, von wem von den Steinen ideologisch beeinflusst wurde und umgekehrt, an wen er seine Ideen weitergegeben hat.

\section{Die Erforschung des Flusses Xingu}

Der Rio Xingu ist durch seine Länge von über $2000 \mathrm{Ki}$ lometern der zweitgrößte Strom Brasiliens. In mehreren Quellflüssen entspringt er an der nördlichen Abdachung des Berglandes von Mato Grosso und mündet mit einer langen und bis zu acht Kilometer breiten Trichterform unterhalb der Gegend von Pôrto de Moz in den Amazonas. Geografisch betrachtet bildet er die natürlichste und gradlinigste Verbindung zwischen der Zentralprovinz Mato Grosso und dem Unterlauf des Amazonasstromes bzw. dem bereits damals wichtigen Handels- und Hafenplatz Pará, dem heutigen Belém. Im Gegensatz zu den anderen bedeutenden südlichen Nebenflüssen des Amazonas war der Verlauf des Xingu so gut wie unbekannt geblieben - obwohl seine Mündung schon zu Beginn des 16. Jahrhunderts beobachtet worden war (Rössler, 1939, 270). Zwar versuchte bereits im Jahre 1843 Prinz Adalbert von Preußen von Pará aus den Fluss aufwärts zu befahren, doch verhinderten unüberwindbare Stromschnellen seine Weiterfahrt. Zudem war besonders das Gebiet nördlich von Cuyabá bislang weitgehend unzugänglich, sodass bis in die 80er Jahre des 19. Jahrhunderts weder der Ursprung noch die Quellen des Flusses Xingu bekannt waren.

Besagte Stromschnellen waren in Verbindung mit dem nur schwer zugänglichen Urwaldgelände auch die Erklärung dafür, weshalb sich in ihrem Schutz indianische Gruppen ungestört von äußeren Einflüssen nach ihren eigenen Traditionen und Lebensvorstellungen erhalten konnten. Weitgehend abgeschirmt und isoliert lebten zu der Zeit der ersten Expeditionen 1883 ungefähr 2500 bis 3000 Bewohner in der Gegend des oberen Xingu, auch Alto Xingu genannt. Diese gehören den vier großen Sprachfamilien der Aruak, Karaiben, Tupí und Gê an, ein anderer Stamm hingegen, die Trumaí, bilden eine eigene Sprachfamilie. Heute wird vermutet, dass alle diese Gruppen ursprünglich aus anderen Gegenden stammten und erst im Laufe des 18. 
Jahrhunderts in dieses Gebiet eingewandert sind. Dennoch wird aber von einer einheitlichen Kultur der Xinguanos, d.h. der Indianer des oberen Xingu gesprochen, da diese verschiedenen Stämme durch äußere Umstände bedingt, einen ähnlichen Anpassungsprozess durchlaufen haben (Hartmann, 1986, 9).

Die Erforschung des Verlaufs des Flusses Xingu blieb Karl von den Steinen und der ihn auf den ersten beiden Expeditionen begleitenden Wissenschaftlern vorbehalten. Innen gelang es zum ersten Mal, bis zu seinen Quellen vorzustoßen, diesen Fluss in seiner ganzen Länge zu befahren und somit einen weißen Flecken auf der Landkarte auszulöschen. Der ursprüngliche Anlass für den Beginn der Forschung in diesem Gebiet war ein wirtschaftlicher: die Anbindung der rohstoffreichen Zentralprovinz Mato Grosso an den Welthandelsplatz Pará. Karl von den Steinen wurde beauftragt, im bisher weitgehend unbekannten Rio Xingu einen schiffbaren Fluss zu finden, der es ermöglichte, Wirtschaftsgüter der Zentralprovinz an den Atlantik zu bringen. Diese Hoffnung erfüllte sich zwar wegen der häufigen und starken Stromschnellen und anderer Hindernisse nicht, die Explorationsreise markierte jedoch den Beginn einer umfangreichen und intensiven deutschen völkerkundlichen Forschung im Bereich des Flusses Xingu, bei der die dort lebenden indianischen Völkerschaften immer mehr in den Mittelpunkt des allgemeinen Interesses rückten. Insgesamt wurden von 1883 bis zum Jahre 1926 sechs deutsche Xingu-Expeditionen durchgeführt, die primär der Erforschung der dort lebenden Indianerstämme und deren Lebensbedingungen galten. 100 Jahre nach der ersten Expedition, im Jahre 1983, wurde unter der Leitung von Günther Hartmann eine siebte Forschungsreise in dieses Gebiet durchgeführt, die es sich zur Aufgabe gemacht hatte, die früheren Forschungsergebnisse zu aktualisieren, indem sie den gegenwärtigen materiellen Kulturbesitz der Indianer und ihre Lebensbedingungen untersuchte, um die Veränderungen zu dokumentieren.

Im Folgenden werden lediglich die ersten beiden Expeditionen, die unter der Leitung von Karl von den Steinen stattfanden, bezüglich ihrer Teilnehmer, ihrer Dauer und inrer Forschungsabsicht genauer dargestellt².

Bei der ersten Expedition Karl von den Steinens ins Xingu-Gebiet im Jahr 1883 wurde er von seinem Vetter, dem Maler Wilhelm von den Steinen und dem Physiker und
Astronomen Dr. Clauss begleitet. Diese Forschungsreise startete in Cuyabá, folgte von dort dem Lauf des Xingu zum Amazonas und endete in Pará. Sie dauerte von April bis Oktober jenes Jahres und galt vor allem der Festlegung und Erforschung des Flusslaufes. Doch sie brachte auch einige völkerkundliche Ergebnisse: Mehrere bisher unbekannte Indianergruppen konnten aufgesucht werden, andere zumindest kurz kontaktiert werden, von wiederum anderen Stämmen erfuhren sie die Namen und ihre ungefähren Wohnsitze. Bedeutsam war hierbei die Tatsache - in den Worten eines Teilnehmers der folgenden Xingu-Expedition - „dass sich hier nicht etwa nur beliebige Stämme, sondern Vertreter der wichtigsten Völkergruppen Brasiliens an dieser gleichsam prähistorischen Stufe erhalten haben", und es so möglich war, "eine neue ethnografische Klassifizierung der meisten bisher bekannten Nationen aufzustellen, ihr Entwicklungszentrum und ihre hauptsächlichsten Verbreitungslinien aufzufinden" (Ehrenreich, 1890, 89).

Als erste Europäer hatten die Teilnehmer dieser Expedition Kontakt mit den Stämmen der Bakairi, Kustenaú, Suyá, Manitsauá und Yuruna, von denen sie auch Sammlungen von Objekten der materiellen Kultur dieser Indianer für das Völkerkundemuseum in Berlin mitbringen konnten. Auf das Herstellen von Fotografien wurde aufgrund der damals noch damit verbundenen Schwierigkeiten verzichtet, stattdessen fertigte Wilhelm von den Steinen zahlreiche Zeichnungen an. Mit dieser ersten Expedition betrat Karl von den Steinen nicht nur geografisches Neuland, sondern warf auch in kulturgeschichtlicher und völkerkundlicher Hinsicht wichtige Fragen und Probleme auf, die von späteren Expeditionen aufgegriffen wurden.

Im Anschluss an diese Expedition entstand sein erstes größeres und bedeutendes Werk (Steinen, 1886), welches allerdings noch hauptsächlich im Stil eines Reiseberichtes gehalten war und nur wenige Kapitel explizit ethnologischen Erkenntnissen widmet.

Von Juli bis Dezember 1887 leitete Karl von den Steinen die zweite Xingu-Expedition, bei der der Rio Kulisehu, ein kleinerer Nebenfluss des Rio Xingu, befahren wurde. Für diese Tätigkeit erhielt er eine wesentliche finanzielle Förderung durch die Humboldtstiftung der Königlich Preußischen Akademie der Wissenschaften zu Berlin (Steinen, 1894, Vorwort), einen weiteren Teil der Kosten übernahm die Carl-Ritter-Stiftung der Gesellschaft für Erdkunde zu 
Berlin ${ }^{3}$. Das Untersuchungsfeld war dieses Mal wesentlich begrenzter als das der ersten Reise. Im Gegensatz hierzu stand die zweite Xingu-Expedition, bei der er wiederum von seinem Vetter Wilhelm von den Steinen, aber auch von dem Berliner Völkerkundler und ehemaligen Mediziner Paul Ehrenreich und von dem Astronomen Paul Vogel begleitet wurde, vor einer Reihe fest umrissener Aufgaben und klar zu formulierender Probleme, die sich aus den Ergebnissen der vorhergehenden Reise ergeben hatten. Diese waren zwar wiederum auch geografischer, in erster Linie aber völkerkundlicher Natur und bestanden aus zahlreichen Erstkontakten mit verschiedenen Stämmen sowie der Dokumentation ihres materiellen Kulturbesitzes. Karl von den Steinens besonderes Interesse galt unter anderem dem Stamm der Bakairi, den er bereits auf der ersten Reise studiert hatte, wobei inn insbesondere ihre sprachliche Stellung innerhalb der Karaiben-Sprachgruppe beschäftigte.

So waren auch die Ergebnisse dieser Expedition ziemlich bedeutend: Neben der Erweiterung der geografischen Kenntnisse über die Provinz Mato Grosso durch kartografische Aufnahmen, Messungen und Bestimmungen, konnten auch umfangreiche Sammlungen, Texte, Grammatiken und andere Materialien von der dort ansässigen indianischen Bevölkerung mitgebracht werden, die wiederum in den Besitz des Berliner Völkerkunde-Museums übergingen.

Ebenfalls Resultat dieser Reise war sein zweites Hauptwerk mit dem Titel Unter den Naturvölkern Zentralbrasiliens, das durch seine topografische Präsentation schon einen wesentlich wissenschaftlicheren Anspruch zum Ausdruck brachte. Es gehört zu den klassischen ethnologischen Reisebeschreibungen und birgt auch einen Reichtum an allgemeinen völkerkundlichen Ansichten sowie Anregungen. Durch die in inm aufgeworfenen Fragestellungen bzw. Forschungsansätze beeinflusste es die nachfolgende vergleichende völkerkundliche Forschung in Brasilien. Inspiriert durch die wissenschaftlichen Ergebnisse dieser Expeditionen und zum Teil auch persönlich ermutigt durch Karl von den Steinen, wurden in den folgenden Jahren weitere Forschungsreisen in dieses Gebiet durchgeführt.

Mit der Erforschung dieser Region war vor dem weiteren Eindringen der europäisch-nordamerikanischen Zivilisation eine noch kaum durch diese beeinflusste Kultur der allgemeinen Wissenschaft zugänglich gemacht worden. Hieraus haben sich neue ethnografische Betrachtungsweisen erge- ben, die nicht nur die Amerikanistik, sondern die völkerkundliche Wissenschaft als solche vor neue Aufgaben stellte. Die deutsche Xingu-Forschung hat eine Reihe für diese Gebiete neuer völkerkundlicher Fragestellungen aufgeworfen, die fruchtbaren Boden für weitere Forschungsreisen und wissenschaftliche Untersuchungen boten (Rössler, 1939, 279).

Da Karl von den Steinen jedoch trotz dieser wissenschaftlichen Pioniertätigkeit im Xingu-Gebiet heutzutage über keinen großen Bekanntheitsgrad verfügt, seien vor einer weiteren Beschäftigung mit seiner Forschung hier zunächst einige biografische Informationen zu seiner Person gegeben.

\section{Zur Person Karl von den Steinens}

Karl von den Steinen wurde am 7. März 1855 in Mühlheim an der Ruhr geboren und besuchte das Gymnasium in Düsseldorf, an welchem er bereits im Jahr 1871, im Alter von 16 Jahren, das Abitur ablegte (Hartmann, 1986b, 13-15; Plischke, 1929; BAA). Anschließend studierte er Medizin in Zürich, Bonn und Straßburg und promovierte 1875 in Straßburg zum Dr. med. mit seiner Dissertation Über den Anteil der Psyche am Krankheitsbild des Veitstanzes. In Berlin und Wien spezialisierte er sich durch eine Psychiatrieausbildung und arbeitete von 1878 bis 1879 als Hilfs- und Assistenzarzt in der psychiatrischen Klinik der Charité in Berlin.

Nachdem er seine medizinische Staatsprüfung abgelegt hatte, trat er zur Abrundung seiner Studien im Jahre 1879 mit 24 Jahren eine zweijährige Weltreise an, bei der er Einrichtungen zur Pflege Geisteskranker in verschiedenen Ländern - u.a. Mexiko, Kalifornien, Java, Indien, Japan, Ägypten und Polynesien - kennenlernen wollte.

Während seines Aufenthaltes in Honolulu im Frühjahr 1880 traf er zufällig Adolf Bastian, dessen Bekanntschaft er bereits in Berlin gemacht hatte. Diese Begegnung sollte seinen weiteren Lebensweg prägen, indem durch Bastian seine Neugier und sein Interesse für die Welt der Naturvölker, mit denen er bereits während seiner bisherigen Reise in Berührung gekommen war, in systematischmethodische Bahnen gelenkt wurden. Nach seiner Rückkehr von dieser Weltreise begann er folglich sich intensiver mit

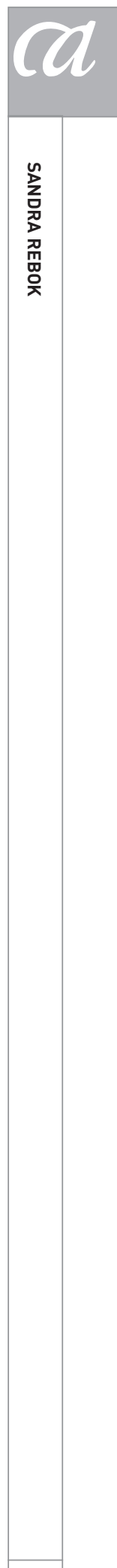

203 
der Völkerkunde zu beschäftigen und trat der Berliner Gesellschaft für Anthropologie, Ethnologie und Urgeschichte bei. Durch diese Weltreise war in inm die Begeisterung für die Tätigkeit als Forschungsreisender erwacht, die inn 1882 veranlasste, als Naturforscher und Arzt an der Deutschen Antarktisexpedition teilzunehmen, bei der vorwiegend Beobachtungen über das Leben der Robben und Vögel vorgenommen wurde.

Nach Beendigung dieser Expedition im Jahr 1883 lösten sich Karl von den Steinen und Otto Clauss in Montevideo, Argentinien, von den anderen Mitgliedern der Expedition, um gemeinsam mit dem Maler Wilhelm von den Steinen eine Expedition im zentralen Südamerika durchzuführen. Nach brieflicher Kontaktaufnahme mit Adolf Bastian, der zu dieser Zeit Direktor des Museums für Völkerkunde in Berlin war, wurde hierfür Zentralbrasilien als Ziel ausgewählt. So kam es 1884 zur ersten deutschen Xingu-Expedition, die für von den Steinen die erste völkerkundliche Forschungsreise darstellte, und somit das Ergebnis seiner neuen Orientierung war. Drei Jahre später führte er wesentlich besser ausgerüstet und vorbereitet die zweite Forschungsreise in der Region durch, die eine Fortsetzung seiner bisherigen Studien zum Ziel hatte.

Im Jahr 1889 habilitierte Karl von den Steinen an der Friedrich-Wilhelms-Universität in Berlin für Völkerkunde mit einer Arbeit über Erfahrungen zur Entwicklung eines Völkergedanken, wobei in diesem Zusammenhang auch der ethnografische Teil seines zweiten Werkes Durch Centralbrasilien bewertet wurde 4 .

Neben seinem Interesse für die Ureinwohner Brasiliens faszinierte inn bereits seit seiner Weltreise die Inselwelt Polynesiens, daher kam inm ein Auftrag des Berliner Völkerkundemuseums, die Sprache, Kunst und Kultur der Marquesaner in Polynesien einer eingehenden Untersuchung zu unterziehen, sehr gelegen. So reiste er im Sommer 1897 über Kanada, Vancouver, San Francisco zu den Marquesas-Inseln und widmete sich ein halbes Jahr lang der Erforschung der dortigen Kulturen und dem Sammeln von völkerkundlichem Material.

In der Zeit nach dieser Forschungsreise wandte er sich mehr der Arbeit im institutionellen Rahmen zu. Er wertete seine bisherigen Forschungsergebnisse aus und unternahm außer einer letzten Reise 1902 in die Vereinigten
Staaten keine weiteren Expeditionen mehr. Im Jahr 1900 wurde er sowohl zum Abteilungsleiter der amerikanischen Sammlungen am Völkerkundemuseum in der Nachfolge Eduard Selers ernannt als auch zum außerordentlichen Professor an der Friedrich-Wilhelms-Universität in Berlin. In der entsprechenden Akte des Ministers der geistlichen, Unterrichts- und Medicinal-Angelegenheit vom 6. Februar 1906 wird hierbei als sein Aufgabenbereich benannt, "die Völkerkunde mit besonderer Berücksichtigung der Ethnologie in Vorlesungen und soweit erforderlich auch in Übun-

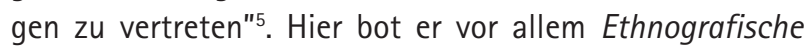
Übungen im Königlichen Museum für Völkerkunde, privatissime und unentgeltlich an, aber auch eine Vorlesung über die Polynesische Mythologie ${ }^{6}$. Ebenfalls ab 1900 und bis 1911 war er stellvertretender Vorsitzer der Berliner Gesellschaft für Anthropologie, Ethnologie und Urgeschichte und erhielt im Jahr 1904 die Stelle des Direktors der südamerikanischen Abteilung des Museums.

Im selben Jahr wurde er allerdings wiederum von seinen Lehrverpflichtungen an der Universität entbunden, was offiziell mit seinen vielen Reisen und mit seiner Tätigkeit am Museum begründet wurde, die von der Fakultät als der Lehre hinderlich angesehen wurden ${ }^{7}$. In Wirklichkeit geschah dies aber auf den Wunsch Karl von den Steinens hin. Trotzdem hielt er aber weiterhin seine Ethnografischen Übungen und zudem eine Veranstaltung über die Ethnografie der Naturvölker Südamerikas mit Demonstrationen am Museum für Völkerkunde $a b^{8}$. Die Tätigkeit in einer verbeamteten Stellung entsprach offenbar weniger seinen Neigungen und so zog er sich bereits 1906 von allen öffentlichen Ämtern zurück, um sich ausschließlich als Privatmann der wissenschaftlichen Arbeit zu widmen. Ab dieser Zeit arbeitete er hauptsächlich an der Auswertung und Publikation seiner Studien über die Marquesas, wobei er sich insbesondere hiermit unter dem künstlerischen Aspekt beschäftigte. Ergebnis war die Veröffentlichung einer völkerkundlichen Monografie über die Marquesas-Inseln, die 1925/28 in zwei umfangreichen Bänden und einem Bilderatlas unter dem Titel Die Marquesaner und ihre Kunst erschien.

Weiterhin regen Anteil nahm er an dem Wirken der Berliner Gesellschaft für Anthropologie, Ethnologie und Urgeschichte, deren Vorsitz er von 1908 bis 1910 übernahm und die inn 1925 anlässlich seines 70. Geburtstages zum Ehrenmitglied ernannte. Ein Jahr später wurde er ebenfalls 
zum Ehrenmitglied der Société des Américanistes de Paris ernannt. Am 4. November 1929 verstarb Karl von den Steinen im Alter von 75 Jahren in Kronberg im Taunus durch einen am gleichen Tag erlittenen Schlaganfall.

\section{Bedeutung Karl von den Steinens für die damalige deutsche Amerikanistik: Bezug zWischen Makro- und Mikroebene}

Im Folgenden wird der Bezug hergestellt zwischen der dargestellten Makro- und Mikro-Ebene, das heißt, die Arbeit Karl von den Steinens wird in den historischen Kontext der damaligen Amerikanistik in Deutschland gestellt. Dies gibt zum einen Aufschluss darüber, welche Verbindung zwischen seinem Anliegen bzw. seiner Forschungsmethode und den zeitgenössischen Fragestellungen bzw. wissenschaftlichen Vorgehensweisen zu sehen sind und zum anderen, was als sein persönlicher Beitrag in der deutschen Amerikaforschung bezeichnet werden kann. Mit anderen Worten, es soll dargelegt werden, in welcher Hinsicht Karl von den Steinen mit seiner wissenschaftlichen Arbeit dem damaligen Zeitgeist entsprach und an welchen Stellen er Innovationen herbeiführte.

\section{III.1. Traditionelle Aspekte der Forschung Karl von den Steinens}

Bei der Untersuchung derjenigen Aspekte, mit denen Karl von den Steinen in der Tradition der damaligen ethnologischen Forschung steht, ist zunächst - als offensichtlichste Auswirkung seiner Zeit - seine evolutionistische Sichtweise zu nennen (Thieme, 1993, 68-76). Ein dringendes Anliegen der damaligen Völkerkunde war, „bis an die Wiege des Menschentums vorzudringen, um im Hinblick auf die Entwicklung der Daseinsformen menschliche Wesen besser zu verstehen" (Schaden, 1956, 276). Hierfür konnten aber nur solche Stämme aufschlussreiche Informationen geben, die noch nicht durch das Zusammentreffen mit der außen stehenden Zivilisation ihre „naturvölkische Eigenart" eingebüßt haben. Die Indianer-Stämme des Xingu-Gebietes boten nach diesen Kriterien gute Voraussetzungen für eine solche Art der Untersuchung und Karl von den Steinen studierte jene Stämme auch in der Überzeugung, in ihnen die ursprünglichste Stufe auf der Skala der menschlichen Entwicklung zu finden. Hierbei war er bestrebt, sowohl auf die weitere Entwicklung dieser Völker als auch die Ur- sprünge der eigenen Kultur Rückschlüsse ziehen. Folglich waren viele seiner Fragestellungen und Forschungsansätze von diesem Gedankengut durchzogen.

Des Weiteren interessierte er sich für die in jener Zeit äußerst aktuellen historisch ausgerichteten Fragestellungen nach der Geschichte und dem Ursprung der Völker als auch nach den vollzogenen Völkerwanderungen und der Herkunft einzelner Stämme.

Seine im Zuge seiner Habilitation erfolgte Auseinandersetzung mit der Theorie der Elementar- und Völkergedanken ist ebenfalls im Zusammenhang mit der ideologischen Strömung jener Epoche zu sehen.

Auch die Art des Reisens und der personellen Ausstattung seiner Expeditionen ging einher mit den Gepflogenheiten seiner Zeit. Unter militärischem Geleitschutz zog er von Dorf zu Dorf, wobei es kaum zu längeren Aufenthalten bei einem Stamm kam. Es handelte sich also mehr um eine Forschungsreise als um eine Feldforschung im modernen Sinne.

Seine Untersuchungen konnten daher auch nicht sehr in die Tiefe gehen, sondern richteten den Fokus meist auf den Vergleich möglichst vieler Ethnien. Dies entsprach ebenfalls der Art der damaligen ethnografischen Studien: In der Regel waren dies groß angelegte Untersuchungen einer bestimmten Region mit der Zielsetzung, eine erste Übersicht über die in ihr vorhandene indigene Welt zu erlangen.

Auch sein Bestreben, die materielle Kultur der besuchten Dörfer zusammenzutragen und nach Deutschland zu bringen, ist im zeitgenössischen Kontext zu sehen. Man war sich der Tatsache bewusst, dass es in kürzester Zeit zu bedeutenden Veränderungen bei der indianischen Bevölkerung kommen würde - sei es durch das Aussterben der Stämme oder durch die Assimilation dieser - und man wollte jede Information über sie für die Nachwelt retten. Aus eben diesem Grund rief Karl von den Steinen an mehreren Stellen selbst zum Erforschen der noch unbekannten Indianerstämme Südamerikas auf (Steinen, 1895, 249).

Auch sein Vorgehen beim Sammeln dieser Objekte unterschied sich nicht grundlegend von der damals gängigen Methode: Einen Teil erwarb er durch Tausch, einen anderen eignete er sich hingegen einfach an, wenn die Besitzer vor inm in den Wald geflüchtet waren. 
In diesem Zusammenhang müssen ebenfalls seine anthropologischen Vermessungen ${ }^{9}$ und auch seine Sprachstudien (Steinen, 1886; 1892; 1904) genannt werden. Mit beiden Ansätzen versuchte er, dem in der damaligen Ethnologie vorrangigen Forschungsanliegen einer Klassifikation der Indianerstämme nachzugehen (Thieme, 1993, 56-61).

\section{III.2. Innovative Aspekte der Arbeit Karl von den Steinens}

Die ethnologischen Ergebnisse seiner beiden Xingu-Expeditionen haben in den letzten beiden Jahrzehnten des 19. Jahrhunderts eine neue Epoche für die Indianerforschung in Südamerika eröffnet ${ }^{10}$, da sich von den Steinen nicht mehr damit begnügte, mehr oder weniger zufällige Informationen zusammenzutragen, sondern zielgeleitet die Durchforschung bestimmter Problemkomplexe ins Auge fasste. Auch seine Methode, das Aufgefundene zu verarbeiten und dabei größere Kulturzusammenhänge aufzurollen sowie sein komparativer Ansatz gaben der südamerikanischen Völkerkunde neue Anregungen.

So gaben seine Beobachtungen soziologischer und völkerpsychologischer Art der damaligen ethnologischen Forschung neue Impulse:

„Im Gegensatz zu der bisher geübten Form, Indianergruppen vor allem sprachlich zu definieren und zu gliedern, begann nun Karl von den Steinen, sich ausführlich und intensiv sowohl mit dem materiellen Kulturbesitz der besuchten Gruppen zu befassen als auch den geistigen Kulturbereich mit einzubeziehen und soziologische und völkerpsychologische Aspekte dabei nicht außer acht zu lassen" (Hartmann, 1986b, 42).

Im Zusammenhang hiermit ist sein Interesse für das Studium der Mythen, Märchen und Legenden der Xingu-Indianer zu sehen. Die Erforschung dieser sogenannten „oralen Literatur" der sogenannten Naturvölker war ein Aspekt der ethnologischen Arbeit, der zu der betreffenden Zeit gerade im Entstehen begriffen war und maßgeblich durch die Untersuchungen Karl von den Steinens vorangetrieben wurde. Kaum je zuvor ist in der ethnologischen Literatur der "Sagenschatz eines Eingeborenenstammes so geistreich für die Interpretation der sich darin spiegelnden Weltanschauung ausgewertet worden" (Schaden, 1956, 276), wie es Karl von den Steinen mit der Ahnensage und anderen mythischen Überlieferungen der Bakairí getan hat.
Bei Fragestellungen, bei denen inm die psychologisch ausgerichtete Völkerkunde nicht weiterhelfen konnte - wie beispielsweise bei dem Problem der Herkunft des Volksstammes der Karaiben - ging er bei seinen Untersuchungen wiederum auch kulturhistorischen und linguistischen Zusammenhängen nach. Seine linguistischen Arbeiten sind zwar als solche keine Innovation, jedoch wurden zuvor kaum derartig systematische, fundierte und wissenschaftlich ausgerichtete Sprachstudien durchgeführt.

Als innovativ kann zudem auch der bereits relativ reflektierte Zugang bezeichnen werden, den Karl von den Steinen zur Ethnologie bzw. ethnografischen Forschung an den Tag legte. In seinen Werken wies er des öfteren auf Schwierigkeiten hin, die sich bei seinen Untersuchungen ergeben hatten, und gab Arbeitsanleitungen sowie Ratschläge für künftige Studien ${ }^{11}$. Des Weiteren zeigte er die Fehler seiner eigenen Arbeit als auch bei früheren Studien anderer Forscher auf und warnte davor, dieselben zu wiederholen. Bei der Beschreibung der indigenen Welt fügte er immer wieder Vergleiche aus der eigenen Kultur bei, um die Darstellungen verständlicher zu machen und letztendlich auch zu relativieren. Nicht zuletzt zeichnet sich seine Arbeit auch durch die Tatsache aus, dass er ein relativ positives, realistisches Bild von den Indianern hatte, das - abgesehen von seiner evolutionistischen Überzeugung - verhältnismäßig wenig durchzogen war von ideologisch verklärten Vorstellungen.

Zusammenfassend lässt sich sagen, dass Karl von den Steinens Forschung eine nicht zu unterschätzende Bedeutung für die deutsche Amerikanistik jener Zeit hatte, was sich auch darin manifestiert, dass einer der meist gesehenen und meist beachteten Wissenschaftler Deutschlands auf den Internationalen Amerikanistenkongressen war. Durch seine beiden Xingu-Expeditionen und deren Auswertung wurde er zu einer bedeutenden Persönlichkeit innerhalb der Brasilienforschung. Seine beiden Hauptwerke gehören zu den klassischen Werken der Völkerkunde Brasiliens, machten ihn in weiten Kreisen bekannt und trugen nicht zuletzt auch zur Popularität der Völkerkunde in Deutschland bei. Dies lag nicht zuletzt an seinem allgemein verständlichen und klaren Stil sowie an seiner ungekünstelten, humorvollen Beschreibung der verschiedenen Begebenheiten auf seinen Expeditionen. Wie Egon Schaden es ausdrückt, durch diese:

„Art der Darstellung, die sich nie unnötig im Bereich abstrakter Erwägungen verlor und die sowohl bei der Schil- 
derung der Reiseerlebnisse als auch bei der Behandlung wissenschaftlicher Themen stets den warmen Indianerfreund erkennen ließ, ist es Karl von den Steinen wie kaum einem anderen Ethnologen um die Jahrhundertwende gelungen, nicht nur bei Fachgenossen, sondern ebenso beim Laienpublikum zahlreiche begeisterte und dankbare Leser zu finden" (Schaden, 1956, 278).

In der Tat war die erste Ausgabe seines Werkes Unter den Naturvölkern Zentralbrasiliens schnell vergriffen und aufgrund der weiteren Nachfrage wurde umgehend eine billigere "Volksausgabe" herausgegeben.

Als letztes Beispiel für die Bedeutung, die Karl von den Steinen für die deutsche Amerikanistik hatte, sei die Tatsache genannt, dass im Xingu-Gebiet ein Fluss nach inm benannt worden ist.

\section{Aktuelle Gültigkeit und Relevanz der Forschung Karl von den Steinen}

Wie bereits erwähnt, ist trotz der Bedeutung, die Karl von den Steinen für die deutsche Amerikanistik und insbesondere natürlich die Brasilienforschung gehabt hat, sein Name heute eher unbekannt. Was auf den ersten Blick vielleicht wie ein Widerspruch aussehen mag, lässt sich relativ leicht erklären. Die Ethnologen des 19. Jahrhunderts, die heute noch allen geläufig sind, sind vor allem diejenigen, die neue Theorien aufgestellt, innovative Forschungsansätze verfolgt und Grundlagenwerke der Ethnologie verfasst haben bzw. in der Lehre tätig waren oder wichtige Positionen innehatten.

Karl von den Steinen hat zwar eine wichtige Pionierleistung vollbracht - bei seiner Forschung ist er mit einem bedeutenden wissenschaftlichen Ansatz vorgegangen und hat für seine Zeit eine recht fortschrittliche Methodik verwendet - aber seine Forschung war regional sehr begrenzt. Daher ist er hauptsächlich innerhalb der ethnologischen Forschung dieser speziellen Region bekannt. Zudem hat er sich auch weder in bemerkenswerter Weise der Lehrtätigkeit gewidmet noch umfassende ethnologische Theorien entwickelt, was ebenfalls dazu beiträgt, dass er außerhalb der Xingu-Forschung nur selten erwähnt wird.
Heißt dies aber auch zwangsläufig, dass Karl von den Steinens Forschung heutzutage keine Bedeutung mehr hat? Sind die Resultate seiner Studien inzwischen widerlegt worden oder haben sie immer noch Gültigkeit? Um der Beantwortung dieser Fragen nachzugehen, wird im Folgenden etwas weiter ausgeholt.

Ein Charakteristikum der ethnologischen Forschung des 19. Jahrhunderts war die Vorstellung, dass es eine objektive Realität gibt und man lediglich die richtige Methodik finden musste, um diese zu erfassen. Daher bestand ein Teil des ethnologischen Diskurses darin - wie übrigens in den anderen wissenschaftlichen Disziplinen auch - die angemessene Methode hierfür zu definieren. Heute hingegen wird die Idee, die Realität völlig objektiv kennenzulernen, ausgeschlossen. Der Grund hierfür ist die Tatsache, dass nicht mehr von der Existenz einer einzigen Realität ausgegangen wird, sondern von der Überzeugung, dass es ebenso viele "Wirklichkeiten" gibt, wie Arten sich dieser anzunähern oder Personen, die diese zu beschreiben versuchen. Gemäß der Orientierung des Forschers sowie dem seinen Untersuchungen zugrunde liegenden Paradigma, erhält man die eine oder andere Vision der angetroffenen Realität. Ein vollkommenes Verstehen und Begreifen dieser ist vor allem dann unmöglich, wenn es sich - wie es bei der Ethnologie der Fall ist - um soziale Tatsachen handelt. Die Forschung ist keine einfache Beschreibung mehr, sie hat sich vielmehr in eine Diskussion verwandelt, bei der man über die von der Ausrichtung der Untersuchung abhängende wahrgenommene Wirklichkeit spricht.

Aus diesen Überlegungen heraus lässt sich schlussfolgern, dass eine fundierte Untersuchung immer eine gewisse Gültigkeit beibehält, auch wenn der ihr zugrunde liegende Ansatz nicht mehr aktuell ist oder spätere Forschungen zu anderen Ergebnissen gekommen sind. Vielmehr sind die Qualität der Untersuchung und ihre Methodik ausschlaggebend, um die Nützlichkeit und Relevanz der betreffenden Arbeit zu bestimmen.

Des Weiteren sollte man sich veranschaulichen, dass ein konkretes Forschungsergebnis, wie es zum Beispiel das Erstellen einer Klassifikation ist, immer ein künstliches Konstrukt bleibt und ein gewisses Fehlerpotenzial in sich birgt, da man die Realität notwendigerweise einem Schema zuordnet.

ARBOR CLXXXV 735 enero-febrero [2009] 199-213 ISSN: 0210-1963

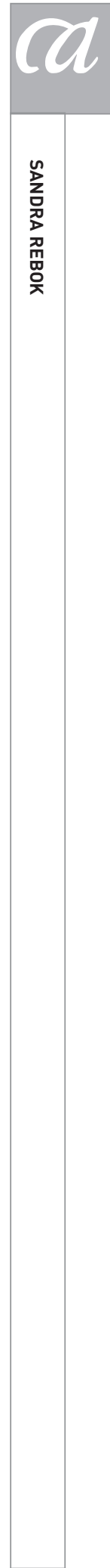


Was bedeutet diese Sichtweise nun für die Evaluierung der wissenschaftlichen Arbeit Karl von den Steinens? Zunächst einmal, dass bei der Frage nach der Relevanz seiner Forschung der Schwerpunkt nicht auf der Überprüfung seiner konkreten Forschungsergebnisse liegen sollte. Wichtiger als die Suche nach inzwischen widerlegten Ansichten, nach Fehlern in seinem Denken oder in seinen Schlussfolgerungen ist es, die immer noch gültigen Ansätze seiner Forschung herauszustellen. Manche seiner Resultate sind mit Sicherheit heute überholt. Andere wiederum lassen sich nicht einmal mehr überprüfen, da die betreffenden Ethnien inzwischen ausgestorben sind. Letztendlich lässt sich nicht immer mit Bestimmtheit sagen, dass ein Ergebnis nachweislich falsch ist, auch wenn man heute zu anderen Schlussfolgerungen gekommen wäre. Und wer kann darüber hinaus denn versichern, dass das aktuelle Ergebnis richtig ist und man nicht in der Zukunft mit wiederum neuen Methoden ein anderes Resultat erzielen könnte?

Unter diesen Umständen erscheint es daher wesentlich angemessener, sich bei der Frage nach der Relevanz der Forschung Karl von den Steinens statt der Resultate vielmehr seine spezifische Herangehensweise an fremde Völker und seine Forschungsmethodik genauer zu betrachten. Einige Aspekte dieser haben heute immer noch Gültigkeit, andere wiederum werden aus heutiger Sicht nicht mehr als adäquat angesehen oder aber lediglich nicht mehr in dieser Weise praktiziert.

Widmen wir uns zunächst denjenigen Aspekten seiner Forschung, die auch bei der aktuellen Südamerikaforschung immer noch von Bedeutung sind.

Karl von den Steinen interessierte sich für das geistige Leben der Xingu-Indianer, also für diejenigen Bereiche der Kultur, die Aufschluss über das Denken der untersuchten Ethnien und über ihr Wesen geben. Er versuchte unter anderem mit Hilfe der Mythen, der Märchen sowie der Sagen zu dem kollektiven Unterbewussten dieser Menschen zu gelangen und versprach sich von der Analyse ihrer oralen Kultur, einen Einblick in ihr Denken zu erhalten. Diese psychologische Ausrichtung der Forschung - der Versuch, die Persönlichkeit von Individuen anhand der Kultur, in der sie leben, zu analysieren - ist ein sehr aktueller Ansatz.

Von großer Bedeutung für die heutige Amerikanistik sind ebenfalls seine Sprachstudien. Diese basieren auf der Über- zeugung, dass die Xingu-Stämme untereinander verschieden sind und sich diese Unterschiedlichkeit in der Sprache manifestiert. Daher analysierte er diese Differenzen mit dem Ziel, die Xingu-Indianer anhand ihrer Sprache zu klassifizieren. Auch solche Untersuchungen werden heute noch durchgeführt, wenn auch nicht mehr unter denselben Bedingungen. Bei neuen Versuchen einer Klassifikationen der Xingu-Stämme muss man auf frühere Sprachaufzeichnungen zurückgreifen - und hierbei eben vor allem auf die von den Steinens -, da sich inzwischen zum einen die Zahl dieser Volksgruppen drastisch reduziert und zum anderen die Sprache der Überlebenden sich unter äußeren Einflüssen merkbar gewandelt hat. Allerdings soll hier nochmals darauf hingewiesen werden, dass eine Klassifikation lediglich ein Versuch sein kann, mittels bestimmter Kriterien Ordnung in eine Gesamtheit zu bringen und keine absolute Wahrheit darstellt. Auch heute noch werden daher ständig Zugehörigkeiten hinterfragt und neue Vorschläge für eine modifizierte Einteilung unterbreitet.

Ein weiterer Aspekt seiner Sprachforschung - ebenfalls von Aktualität - ist sein Interesse für den Zusammenhang von Sprache und dem Denken sowie der Geistesverfassung der jeweiligen Ethnie. Karl von den Steinen untersuchte, inwieweit die Sprache das Denken beeinflusst bzw. Aufschluss hierüber gibt (Steinen, 1894, 81), also Fragestellungen, die den linguistischen Zweig innerhalb der heutigen Ethnologie noch immer beschäftigen.

Nachdem wir gesehen haben, in welchen Aspekten Karl von den Steinens Werke immer noch relevant sind, stellt sich nun die Frage, worin sich im Gegensatz hierzu sein Ansatz von dem der heutigen Amerikanistik unterscheidet.

In erster Linie besteht dieser Unterschied vor allem darin, dass heute derartig groß angelegte vergleichende Forschungen nicht mehr durchgeführt werden. Karl von den Steinens Aufgabe war es, eine erste Übersicht über die bis dahin unbekannten, im Xingu-Quellgebiet lebenden Indianer-Stämme, zu erstellen. Heute hingegen sind diese Stämme bekannt und "ethnografisch erfasst". Bei einer erneuten Erforschung der noch existierenden Stämme werden hingegen in der Regel konkrete Aspekte untersucht, eng gefasste Fragestellungen formuliert und lediglich ein kleiner Ausschnitt der vorgefundenen Wirklichkeit analysiert. Meist geht dies auch mit einem längeren Aufenthalt bei einem bestimmten Stamm oder Dorf einher, um das Leben in diesem detaillierter zu erfassen. 
Abgesehen hiervon ist selbstverständlich auch seine evolutionistische Überzeugung überholt. Die ethnologische Forschung geht nicht mehr von dem notwendigen Durchlaufen bestimmter Entwicklungsstufen aus, sondern von einer an die entsprechende Umwelt angepassten Gestaltung der Lebensform. Demnach werden auch keine Studien mehr über fremde Völker durchgeführt, um daraus Rückschlüsse auf bzw. Informationen über den Ursprung unserer eigenen Kultur zu gewinnen.

Als Antwort auf die eingangs gestellte Frage nach der Gültigkeit und der Relevanz Karl von den Steinens studien für die ethnologische Forschung in Südamerika heutzutage lässt sich als Fazit Folgendes sagen: Auch wenn die Gültigkeit seiner Untersuchungen in manchen Aspekten in Frage gestellt werden mag - in anderen wiederum wird ihre aktuelle Bedeutung nicht bezweifelt - so ist die Relevanz seiner Arbeit im Xingu-Gebiet für die aktuelle Brasilienforschung in einer wichtigen Hinsicht über jeden Zweifel erhaben. Karl von den Steinen hat eine damals unbekannte und von der Zivilisation völlig abgeschiedene Welt kennengelernt und untersucht. Er konnte das Leben der Indianer in ihrer "ursprünglichen", von europäischen oder amerikanischen Einflüssen unberührten Ausprägung studieren. Die Aufzeichnungen Karl von den Steinens sind schon alleine aus diesem Grund äußerst wertvoll und von großer Bedeutung für die heutige Forschung, weil sie Informationen darlegen, die heutzutage nicht mehr eingeholt werden können. Dies gilt sowohl für die ethnografischen Beschreibungen der betreffenden Völker als auch für die durchgeführten Sprachaufnahmen. Wer sich heutzutage - aus welchem Grund auch immer - für die Welt der Xingu-Indianer interessiert, wer ihre Herkunft, ihre Verwandtschaftsverhältnisse untersuchen will, muss zwangsläufig auf die Werke Karl von den Steinens zurückgreifen. Schon diese Tatsache allein lässt seine Werke zu einem wichtigen und unverzichtbaren Beitrag zur aktuellen Südamerikaforschung werden.

\section{EINflüsSE Von UNd AUf ANDERE VölKeRKUndLeR}

Sowohl um einen Wissenschaftler in seinen zeitgeschichtlichen Kontext einzuordnen, als auch um die Bedeutung seiner Forschung besser einschätzen zu können, ist es aufschlussreich zu untersuchen, wessen Ideen ihn bei seiner Arbeit beeinflusst haben und wem er wiederum selbst als Inspiration diente. Betrachten wir daher nun genauer die Urprünge der ethnologischen Studien Karl von den Steinens, bei wem er seine Ausbildung hierzu erhielt, von wem er ideologisch beeinflusst wurde und auf wen er sich selbst bei seiner Arbeit bezog.

Wie bereits erwähnt, begann Karl von den Steinen seine Laufbahn als Ethnologe im Jahr 1880, nachdem er in einem Hotel in Hawaii Adolf Bastian begegnet war (Köpping, 1983, 23). Es war Bastian, der sowohl seine Begeisterung für die völkerkundliche Forschung geweckt, als auch inn weiterhin ideologisch beeinflusst hat. Dafür spricht zum einen die Tatsache, dass von den Steinen im letzten Viertel des 19. Jahrhunderts unter Bastians Leitung am Museum für Völkerkunde in Berlin gearbeitet hat, und zum anderen belegt auch das Thema seiner Habilitationsschrift seine Auseinandersetzung mit Bastians Theorien. Des Weiteren erwähnt er mehrmals die von Bastian geforderte Dringlichkeit bei der Erforschung der Naturvölker, bevor diese assimiliert oder gar ausgestorben seien. Sein Interesse am Zusammentragen der materiellen Kultur der betreffenden Völker kann mit Sicherheit entweder dem ideologischen Einfluss Bastians oder aber direkt den diesem in seiner Funktion als Direktor des Museums gegebenen Instruktionen bei der Feldforschung zugeschrieben werden.

Die weitere Beantwortung dieser Fragestellung gestaltet sich hingegen als eher schwierig. Es liegt in der Natur der Sache, dass Wissenschaftler in den wenigsten Fällen offen darlegen, wer sie ideologisch beeinflusst hat, wessen Ideen sie aufgreifen und weiterentwickeln. Dies ist bei Karl von den Steinen nicht anders. Auch in der Sekundärliteratur über sein Wirken sind kaum direkte Vermerke zu diesen Fragestellungen zu finden.

Daher ist es gerade die Aufgabe bzw. die Herausforderung der Forschung, Einflüsse auf einen Wissenschaftler zu entdecken, indem man seine Werke kennt, über seine Biografie informiert ist und auch über die ideologische Ausrichtung derjenigen Personen bescheid weiß, mit denen er beruflich Kontakt hatte.

Im Folgenden werden einige Möglichkeiten dargelegt, um den genannten Zusammenhängen näher zu kommen. Diese Hinweise sprechen zwar nicht automatisch für eine Beeinflussung Karl von den Steinens durch die erwähnten

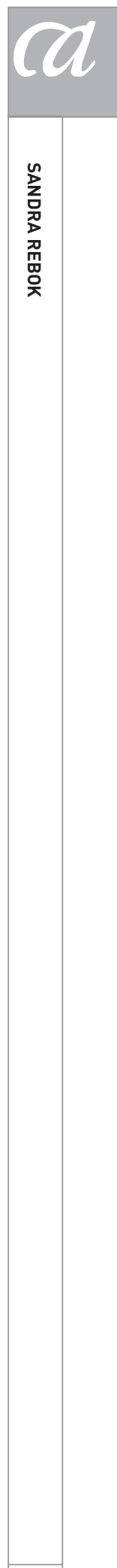

ARBOR CLXXXV 735 enero-febrero [2009] 199-213 ISSN: 0210-1963 
Personen, können aber erste Hinweise auf erfolgte Anregungen, Auseinandersetzungen oder auch übernommene Ideen geben.

Karl von den Steinen legte seine Habilitation im Jahr 1889 an der philosophischen Fakultät der Friedrich-WilhelmsUniversität in Berlin ab. Beurteilt wurde seine Arbeit von Herrn von Richthofen und Herrn Kiepert ${ }^{12}$, der Philosoph Wilhelm Dilthey äußerte allerdings seine gutachterlichen Bedenken hinsichtlich dieser Habilitation ${ }^{13}$.

Ein Blick in das Vorlesungsverzeichnis des Jahrganges 1903/04 bzw. 1905/0614 gibt Aufschluss darüber, wer seine Kollegen während seiner Lehrtätigkeit an der Universität Berlin waren bzw. welche Lehrstühle interagierten: In diesen Jahren lehrten außer ihm Prof. Seler, Prof. Richthofen, Prof. Vierkandt, Prof. von Luschan, Prof. Sieglin und Dr. Ehrenreich.

Bei der Frage nach denjenigen Personen, mit denen er im Laufe seines Lebens korrespondiert hat, lassen sich mehrere Briefe finden ${ }^{15}$ : Unter anderem ein Brief von Balduin Möllhausen (12.06.1904), ein Brief an Max Hirsch (19.02.1914), vier Briefe an Wilhelm Reiss (1886-1887), ca. 30 Briefe und Postkarten an Felix von Luschan (1886-1921), ein Brief an Kurt Breysig (1905), ein Brief an Eduard Seler (9.8.1893), ein Brief an Ernst Haeckel (26.6.1895), ein Brief an Georg Schweinfurth (14.6.1895), ein Brief an Georg Friederici (2.10.1927), 67 Briefe und Karten an Richard Andree (1889-1911), 6 Briefe an Heinrich von Nathusius-Neinstedt (1888-1891), 240 Briefe und Postkarten an den Cotta-Verlag (1888-1898), ein Brief an Dankelmann (7.12.1886) und ein Brief an Pietschmann (21.7.1907).

Auffällig ist hierbei zum einen die häufige briefliche Korrespondenz mit Felix von Luschan und Richard Andree und zum anderen die Tatsache, dass kein Brief von oder an Adolf Bastian darunter ist.

Hinsichtlich seiner Reisegefährten bei den beiden XinguExpeditionen dürfte die Beeinflussung durch den Mediziner Paul Ehrenreich am größten gewesen sein, was vermutlich an der beruflichen Affinität gelegen haben mag und in den verstärkten anthropologischen Fragestellungen während der zweiten Expedition zum Ausdruck kommt.

Generell lässt sich sagen, dass in den Werken von Karl von den Steinens nur selten ein theoretischer oder me- thodischer Bezug auf andere Wissenschaftler zu finden ist. Das lässt sich dadurch erklären, dass es sich bei seiner Forschung um die ethnografische Erkundung eines bisher kaum bzw. gar nicht bekannten Gebietes handelte. Daher konnte er sich nur in wenigen Fällen auf bereits vorhandene Forschungsergebnisse beziehen. Zudem war Karl von den Steinen bekanntlich weniger theoretisch ausgerichtet, also jemand der Thesen aufstellt und diese dann mit den Untersuchungen anderer Forscher vergleicht, sondern inm ging es im Allgemeinen vorrangig um das deskriptive Festhalten des Gesehenen sowie um die weitere Erforschung bestimmter Aspekte dessen.

Hierbei gibt es wohlverstanden einige Ausnahmen, allerdings beschränken diese sich fast ausschließlich auf seine Sprachforschung. Das erklärt sich dadurch, dass er hier bereits auf einige frühere Studien bzw. Sprachaufnahmen zurückgreifen konnte. In seinem 1886 erschienen Werk über seine erste Expedition ins Xingu-Gebiet ist in einem Anhang auch lediglich ein Literaturverzeichnis für seine "Sprachvergleichung" angefügt, jedoch keine allgemeine Bibliografie. In dieser führt er unter anderem die Werke von Prinz Maximilian zu Wied-Neuwied, W.C. von Eschwege, H.C. von der Gabeleutz, J.J. von Tschudi, Carl F.P. Martius, Julius Platzmann, Friedrich Müller und Otto Stoll auf. Auch in seiner Studie über die Sprache der Bakairí erstellte er eine ausführliche Liste, die gleichzeitig den Literaturstand bezüglich der damals vorhandenen Wörterverzeichnisse südamerikanischer Sprachen von 1892 aufzeigt (nicht nur aus dem deutschen Sprachraum) und verglich diese im Folgenden miteinander. Seinem zweiten Hauptwerk Unter den Naturvölkern Zentral-Brasiliens hingegen fügte er gar keine Literaturliste bei, nicht einmal hinsichtlich seiner linguistischen Arbeiten; dies trifft ebenso für die weiteren, von ihm verfassten Artikeln und sonstigen Beiträgen zu.

Bei einer der seltenen Gelegenheiten, in denen er in seinem Text einen Kollegen zitierte, bezog er sich im Zusammenhang mit seinen Ausführungen über das Zeichnen bei den Xingu-Indianern (Steinen, 1894, 245) auf Richard Andree und dessen Aufsatz "Das Zeichnen bei den Naturvölkern", der 1889 in dem Werk Ethnografische Parallelen und Vergleiche veröffentlicht wurde. Dies geht wiederum mit der Tatsache einher, dass er mit Andree einen vergleichsweise intensiven Briefkontakt gepflegt hatte, sodass die Vermutung nahe liegt, dass es hier zu einem fundierteren Gedankenaustausch gekommen war. 
Näher befasste sich Karl von den Steinen nachweislich auch mit den Ideen von Carl F.P. Martius. Bei seinen linguistischen Studien bezog er sich explizit auf dessen Forschungsergebnisse bzw. auf seine Einteilung der brasilianischen Stämme anhand der Guck-Theorie (Steinen, 1886, 290). Diese Klassifikation untersuchte er eingehend, prüfte sie auf ihre Gültigkeit hin und widerlegte sie schließlich.

Nun zum zweiten Teil der Fragestellung: Wen hat Karl von den Steinen wiederum beeinflusst, an wen hat seine Ideen weitergegeben und wer bezog sich nach inm auf seine Forschung?

Da sich Karl von den Steinen wenig mit theoretischen oder allgemeinen, grundlegenden Ausarbeitungen beschäftigte, wird er folglich in der allgemeinen ethnologischen Literatur nur sehr selten erwähnt. Im Zusammenhang mit der völkerkundlichen Brasilienforschung hingegen, oder mehr noch, bei der Xingu-Forschung, erfolgt der Verweis auf inn fast zwangsläufig. Es war es, der die dortigen Stämme in einem von der Zivilisation noch unberührten Zustand antraf und diese beschrieb - dies allein verleiht seinem Werk eine große Gewichtigkeit in diesem Kontext. Daher wird bei solchen Referenzen meist hauptsächlich seine innovative Tätigkeit hervorgehoben, sein Verdienst als Pionier auf diesem Gebiet und somit sein Beitrag zur völkerkundlichen Forschung betont; Weiterverarbeitungen von konkreten Ideen lassen sich hingegen weniger finden.

Eine Ausnahme hiervon stellen wiederum die Resultate seiner linguistischen Arbeiten dar. Diese bilden den Bezugspunkt für nachfolgend unternommene Versuche einer Klassifikation der Xingu-Stämme anhand ihrer sprachlichen Unterschiede. Nicht zuletzt beruht dies auch auf der Tatsache, dass ein Teil dieser Völkergruppen zu späteren Untersuchungszeitpunkten bereits ausgestorben war und man somit zwangsläufig auf Karl von den Steinens Aufzeichnungen zurückgreifen musste.

Da von den Steinen neben seiner geringen Theoriebildung auch keine größere Lehrtätigkeit entwickelt hat, gab es nur wenige Forscher, die man als seine Schüler im engeren Sinne bezeichnen könnte. Dennoch lässt sich aber sagen, dass seine Wirkung über die Universität hinaus erfolgt war. So war ein großer Einfluss unter anderem auch auf solche Personen erkennbar, die nie persönlichen Kontakt mit inm hatten (Nordenskiöld, 1930, 223). Dieser Einfluss manifestierte sich weniger im direkten Übertragen von Ideen, sondern vielmehr dadurch, dass sie durch seine Arbeiten zur völkerkundlichen Forschung allgemein oder bezüglich Zentralbrasiliens im Konkreten inspiriert wurden.

Darüber hinaus ermutigte Karl von den Steinen auch persönlich mehrere Forscher zur Weiterarbeit im Xingu-Gebiet. So ist insbesondere bei denjenigen Wissenschaftlern ein Bezug auf inn zu finden, die seine Erforschung der dort lebenden Indianer weitergeführt haben.

Auf Anregung von den Steinens hin und nach einer Beratung durch inn unternahm Hermann Meyer 1895 eine dritte Expedition in das Xingu-Gebiet mit dem Ziel, die Studien von den Steinens fortzusetzen sowie

"(...) die angrenzenden Gebiete zu erschließen und zu erkunden, inwieweit die eigentümlichen ursprünglichen Kulturverhältnisse, auf die Steinen bei einer Reihe von stammesfremden, noch unberührten Stämmen gestoßen war, sich auch bei den von ihm noch nicht besuchten Völkerschaften dieses Gebietes nachweisen ließen bzw. welchen Abweichungen im Kulturzustand man dort begegnen würde" (Meyer, 1906, 455).

Da von den Steinen nach der vierten, ebenfalls von Meyer geleiteten Expedition, wiederholt zu einer baldigen und gründlichen Forschung und Sammlung im Kulisehu-Kuluene-Gebiet gemahnt hatte (Rössler, 1939, 277), begab sich Max Schmidt im Jahr 1900 auf die fünfte Reise ins Xingu-Gebiet. 1902 veröffentlichte er hierüber seine Arbeit Reiseskizzen aus Zentralbrasilien, in welchem er eingangs erwähnt, dass es die vorhergehenden Xingu-Expeditionen waren, die inn zu dieser Forschungsreise inspiriert hatten, und dass er sich hierfür mit von den Steinens Werk Unter den Naturvölkern Zentralbrasiliens ausgerüstet habe (Schmidt, 1902, 29). Drei Jahre später publizierte er eine erneute Auswertung dieser Reise, die sich, wie der Titel Indianerstudien in Zentralbrasilien (Schmidt, 1905) bereits verdeutlicht, primär mit der Bevölkerung dieses Gebietes befasst. Auch hier betont er, dass die vier früheren XinguExpeditionen die Grundlage für seinen Aufenthalt in diesem Gebiet bildeten, und dass er sich bei seiner Forschung auf Karl von den Steinens Werke Durch Central-Brasilien und Unter den Naturvölkern Zentralbrasiliens gestützt hat (Schmidt, 1905, XIII).

ARBOR CLXXXV 735 enero-febrero[2009] 199-213 ISSN: 0210-1963 
Als Fazit dieser Untersuchung lässt sich feststellen, dass die Betrachtung des Zusammenhanges zwischen der Forschung eines bestimmten Völkerkundlers und der inhaltlichen sowie der methodischen Ausrichtung der Amerikanistik zu der betreffenden Zeit durchaus aufschlussreich ist. Die vorliegende Studie zeigt auf, dass Karl von den Steinen zwar einerseits durchaus verhaftet war in den Geistesströmungen seiner Zeit, und seine Forschung sich in großen Teilen an den damaligen Vorgaben orientierte, er sich aber andererseits auch von diesen kontemporären Ausrichtungen lösen und zahlreiche innovative Ansätze schaffen konnte.

Somit lässt sich an seinem Beispiel belegen, dass die Abhängigkeit der Wahrnehmung von den gesellschaftlichen Rahmenbedingungen zwar eine Konstante des menschlichen Perzeptions- und Urteilsfindungsprozesses ist, diese aber auch immer von einzelnen Forschern in einzelnen Aspekten überschritten werden kann. So bleibt stets die Möglichkeit, die in einem bestimmten gesellschaftlichen Kontext gültigen Paradigmen und Ansätze zu verwerfen und Neues auszuprobieren.

Dieses innovative Vorgehen verleiht den Forschungsresultaten der betreffenden Wissenschaftler auch dann weiterhin eine gewisse Gültigkeit und Relevanz, wenn sich die Rahmenbedingungen oder die aus innen resultierenden theoretischen Orientierungen ändern.

Schlussfolgernd soll nochmals die Wichtigkeit der Betrachtung eines Ereignisses in seinem zeitgenössischen Kontext betont werden. Im thematischen Zusammenhang der vorliegenden Arbeit werden hierbei zwei Gegebenheiten sichtbar: Zum einen verdeutlicht die kontextuelle
Einbettung von Ereignissen die Bedingtheit der ethnologischen Forschung durch die betreffenden Rahmenbedingungen. Zu keiner Zeit war bzw. ist die Wissenschaft von dieser isoliert und vollständig objektiv. Noch weniger war dies in der untersuchten Zeitspanne der Fall, weil die Ethnologie in dieser Phase erst im Entstehen begriffen war, sich noch kein abgegrenztes Berufsfeld mit eigenen Lehrinhalten, mit konkreten Forschungsmethoden sowie -paradigmen oder gar spezifischen Ausbildungsmöglichkeiten entwickelt hatte. Es war vielmehr eine Experimentierzeit, in der sich die wissenschaftlichen Disziplinen konstituiert und voneinander abgegrenzt - aber auch Ideen und Theorien voneinander übernommen haben.

Zum anderen bewirkt aber genau dieser Umstand auch wiederum eine gewisse Freiheit bei Forschungsvorhaben. Die damaligen Völkerkundler hatten einen unterschiedlichen beruflichen Hintergrund und die Forscher interessierten sich meist aus ihrem spezifischen Blickwinkel heraus für die ethnologische Forschung. Dies ließ innen auch mehr Platz für eine eigene Entwicklung und für das Ausprobieren ihres persönlichen Ansatzes, um so ihren spezifischen Beitrag zur Weiterentwicklung der Ethnologie zu schaffen. Wie wir am Beispiel von Karl von den Steinen gesehen haben, hat auch er neben seiner Prägung durch die Bedingungen seiner Zeit die Möglichkeit zur Innovation genutzt. Ein zentraler Aspekt von Wissenschaft ist also letztendlich die Fähigkeit, sich von dem Gegebenen zu lösen, um neue Ansätze zu erwägen und umzusetzen. Der Mut zum eigenen schöpferischen Prozess ist von großer Wichtigkeit, da Kreativität - immer begleitet von einer fundierten wissenschaftlichen Methode - in diesem Sinne die Basis der Weiterentwicklung der Wissenschaft darstellt.

\section{NOTAS}

1 Diese Arbeit wurde im Rahmen eines Forschungsprojektes des Ministerio de Educación y Ciencia (HUM200765125-C02-02/HIST) erstellt, das von Dr. Miguel Ángel Puig-Samper geleitet wird.

2 Falls nicht anders erwähnt, basiert diese Darstellung auf der Übersicht von Hartmann (1986, 39-58).
3 Biographisches Archiv zur Anthropologie (BAA). Information über Karl von den Steinen: Lebenslauf, Veröffentlichungen, Biobibliographisches. Erstellt im Seminar für Völkerkunde der Universität Bonn (1997), S. 2.

4 Siehe Akte zum Habilitationsverfahren Karl von den Steinens (1889), Archiv der Humboldt-Universität Berlin.

5 Siehe betreffende Akte im Archiv der Humboldt-Universität Berlin.

Aceptado: 23 de noviembre de 2008 
6 Siehe Vorlesungsverzeichnis für das Jahr 1903/04, Archiv der HumboldtUniversität Berlin.

7 BAA, S. 2.

8 Siehe Vorlesungsverzeichnis des Jahres 1905/06, Archiv der Humboldt-Universität Berlin.

9 Siehe insbesondere entsprechende Kapitel in seinen beiden Hauptwerken: Karl von den Steinen, Durch CentralBrasilien und Unter den Naturvölkern.

10 Siehe beispielsweise die ausführliche Studie zu seinen Untersuchungen der Ethnie der Bororo (Viertler, 1993).

11 Siehe hierzu das Kapitel „Ein Wink für Reisende" in Steinen, 1886.

12 Siehe Akte Meldung zur Habilitation, Archiv der Humboldt-Universität Berlin.

13 BAA, Lebenslauf Karl von den Steinens.

14 Siehe entsprechendes Vorlesungsverzeichnis, Archiv der Humboldt-Universität Berlin.

15 Auskunft hierüber erteilte die Handschriftenabteilung der Staatsbibliothek zu Berlin, Preußischer Kulturbesitz.

\section{BIBLIOGRAPHIE}

Ehrenreich, Paul (1890): „Mittheilungen über die Zweite Xingu-Expedition in Brasilien", Zeitschrift für Ethnologie, Berlin, Verlag von A. Asher \& Co, S. 89-98.

Gewecke (Frauke 1992): Wie die neue Welt in die alte kam, München, DTV/KlettCotta.

Hartmann, Günther (1986a): „Keramik des Alto Xingu: Zentralbrasilien", Veröffentlichungen des Museums für Völkerkunde Berlin, Neue Folge 42,
Abteilung Amerikanische Naturvölker VII, Berlin, Felgentreff \& Goebel KG.

Hartmann, Günther (1986b): Xingú. Unter den Indianern Zentralbrasiliens, Berlin, Dietrich Reimer.

Köpping, Klaus-Peter (1983): Adolf Bastian and the psychic unity of mankind: The foundations of Anthropology in Nineteenth Century Germany, Queensland, University of Queensland Press.

Meyer, Hermann (1906): „Die Kunst der Xingu-Indianer", Beitrag auf dem 14. IAK 1904 in Stuttgart, Stuttgart, S. 455-473.

Nordenskiöld, Erland (1930): "Nécrologie: Karl von den Steinen", Journal de la Societé des Américanistes, Band 22, Paris, S. 221-227.

Plischke, Hans (1929): „Karl von den Steinen", in Deutsches Biographisches Jahrbuch, Band 11, Stuttgart, Berlin; Leipzig, Deutsche Verlags-Anstalt., 1925-1932.

Rebok, Sandra (2002): „Karl von den Steinens völkerkundliche Studien im Xingu-Quellgebiet Brasiliens Ende des 19. Jahrhunderts", Jahrbuch für Geschichte Lateinamerikas, Nr. 39, Köln, Böhlau, S. 371-393.

Roessler, Gustav (1939): „Der Anteil der deutschen völkerkundlichen Forschung an der Entdeckung und Erforschung des tropischen Südamerika vom Ende des 19. Jahrhundert bis zum Beginn des Weltkrieges", in Hans Plischke (Hrsg.), Göttinger Völkerkundliche Studien, Leipzig, Dieterichsche Universitäts-Buchdruckerei.

Schaden, Egon (1956): „Karl von den Steinens Beitrag zur Brasilkunde", StadenJahrbuch, Beiträge zur Brasilkunde, Band 4, São Paulo, S. 271-283.

Schaden, Egon (1993): „Pioneiros Alemães da Exploração Etnológica do Alto Xin- gu", in Vera Penteado Coelho (Hrsg.), Karl von den Steinen: um século de antropología no Xingu, São Paulo, Editora da Universidade de São Paulo, S. 109-129.

Schmidt, Max (1902): "Reiseskizzen aus Zentralbrasilien", Globus, Band LXXXIL, Braunschweig, S. 29-31.

Schmidt, Max (1905): Indianerstudien in Zentralbrasilien, Berlin, Dietrich Reimer.

Steinen, Karl von den (1886): Durch Central-Brasilien. Expedition zur Erforschung des Schingú im Jahre 1884, Leipzig, F.A. Brockhaus.

Steinen, Karl von den (1892): Zweite Schingú-Expedition 1887-88. Die Bakairi-Sprache: Wörterverzeichnis, Sätze, Sagen, Grammatik, Leipzig, K.F. Köhler.

Steinen, Karl von den (1894): Unter den Naturvölkern Zentral-Brasiliens. Reiseschilderung und Ergebnisse der Zweiten Schingú-Expedition 18871888, Berlin, Dietrich Reimer.

Steinen, Karl von den (1895): „SteinzeitIndianer in Paraguay", Globus, Band 67, Braunschweig, S. 248-249.

Steinen, Karl von den (1904): Diccionario Sipibo, Berlin, Dietrich Reimer.

Thieme, Inge (1993): „Karl von den Steinen: Vida e obra", in Vera Penteado Coelho (Hrsg.), Karl von den Steinen: um século de antropología no Xingu, São Paulo, Editora da Universidade de São Paulo, S. 35-107.

Viertler, Renate B. (1993): „Karl von den Steinen e o Estudo Antropológico dos Bororos", in Vera Penteado Coelho (Hrsg.), Karl von den Steinen: um século de antropología no Xingu, São Paulo, Editora da Universidade de São Paulo, S. 131-151. 